\title{
Revolutionary IoT and Big Data in Healthcare: A Predictive Model Analysis
}

\author{
Ashu' ${ }^{1}$, Rashima Mahajan ${ }^{2}$, Sherin Zafar $^{3}$ \\ \{ashuone@gmail.com, rashima.fet@mriu.edu.in,zafarsherin@gmail.com\} \\ MRIIRS, FET, Faridabad ${ }^{1,2}$, CEST Jamia Hamadard ${ }^{3}$
}

\begin{abstract}
Internet of Things (IoT) is emerging as one of the expeditiously thriving technology with an objective to enable a connectivity between everything and entitles the connected "things" to have an efficacious communication with each other. The appellation "Internet of Things" explicates the technologies and various research areas enabling the Internet to be bestowed into the sphere of physical entities. IoT has an extensive number of application domains mainly Traffic management, Healthcare Monitoring, Security, and Daily life (smart city). The advances in technology have provided with low cost sensory devices and rendered easy communication and buffering of data. The IoT has also revolutionized the modern health care management. IoT innovation in the field of healthcare is a collection of heterogeneous computing devices communicating wirelessly with the help of different apps and devices interconnecting the patients and healthcare givers to diagnose, observe, trace and buffer the essential statistics and other important medical documentation. This paper examines the advancements in health care technologies based on IoT and reviews the role of Big Data Analytics in Predictive Model. The paper also divulges how IoT and Big Data can prove to be a game-changer in the field of healthcare and how it is metamorphosing the healthcare management by enhancing the efficiency, reducing the costs involved and streamlining effective patient care.
\end{abstract}

Keywords: IoT, Big data, Health care, Sensors, Applications

\section{Introduction}

One of the most miraculous technological advancements, greatly transforming human lives, and their way of doing things is, Internet. Internet has covered various fields such as Electronic mode of Communication, Online Education, Online Businesses, Social Networking, Online Shopping, and E-Commerce. Internet is now manifesting the world with a modish 
ecosystem, called Internet of Things (IoT). The IoT [1] is a system composed of physical devices assigned with an IP address and capable of collecting and exchanging data with other physical devices. Fig 1. Depicts an IoT System, where all the physical devices are connected and are able to analyse the data recorded and predict based on the existing data.

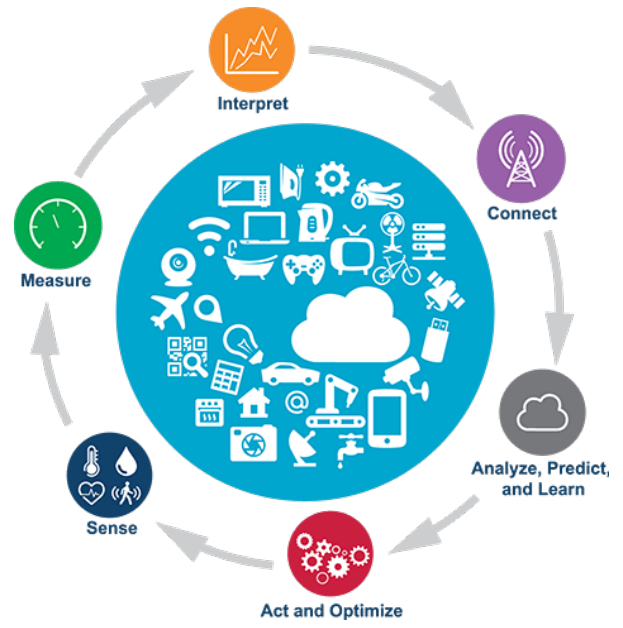

Figure 1. IoT System

IOT is conceptualized on sensors [2] and network enabling the communication and for accessing the data collected. IOT offers to be benevolent in many fields but one of the most prominent areas is the field of health cognizance [3]. The potentiality of IoT has accomplished the development of a large number of medical applications in the field of remote monitoring of health, Supply chain, chronic diseases monitoring, clinical trials and elderly care. The next section of this paper details how the IoT evolution has transformed the healthcare and how it is benefitting the society.

\section{IoT in Health Management:}

IoT devices find an alluring role in the field of Health management. One of subarea is remote health monitoring, where IoT can be used to embed smart devices to observe the vital statistics of the patient such as blood pressure, heart rate, pace makers etc. It can also be used for emergency notification systems, where an emergency beacon is transmitted to the doctor with details about the patient's current situation. Amongst the other applications, another worthwhile medical application is constant assessment of medical condition of senior citizens, ensuring their conventional well-being and providing other disease related assistance. Extensive researches on IoT platform have evolved extensive end-to-end applications [4] for health monitoring related to chronic diseases, prove to be a breakthrough in organizing the recurring medication 
needs. Internet of Things (IOT) thus can also be utilized to produce up to date information to be recorded and saved to be analysed for future use, thus, aiding in generating the effectiveness of a particular medicine under certain medical circumstances. The fig 2. Demonstrates the vital statistics of a patient being monitored and transmitted to the doctor in the hospital and emergency beacons communicated to the ambulance in case of any dire needs.

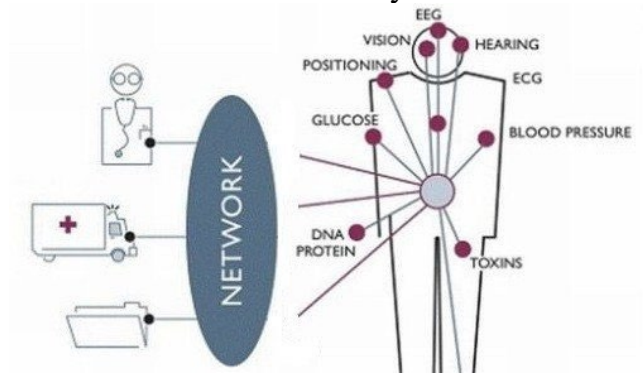

Figure 2. IoT- based Health Care Management System.

The next section of this paper studies the previous researches in the field of Healthcare Management.

\section{Literature Survey:}

The authors in the paper [5] have ventured a blueprint for an intelligent healthcare system based on IoT. The proposed work utilizes a microcontroller to gather the information based on the temperature of the patient and another parameter called the heart rate of the concerned patient and after collecting the information, transmits it through the wi-fi. The system also incorporates an LCD monitor for the patient to be aware of its own health condition. A beacon service is also interspersed in the system by alerting the doctor with the help of a messaging system and warns the care giver.

The authors in paper [6] have evaluated the distinct features of various research activities centered around the IoT based healthcare management systems. It involves survey on the researches carried out for helping the senior citizens, new born (neonatal), patients with chronic diseases, and everyday fitness etc. It also elaborates the various security challenges faced while dealing with the IoT system and the system needs significant research on security grounds.

The authors in paper [7] unwrap the prominence of the Healthcare by involving IOT framework. The focal point of the paper is the health of the people belonging to the remote areas faced by unavailability of doctors. The IOT proves to be the lifesaving mechanism under such conditions. The proposed prototype gathered data from the sensors and synchronized it to the cloud and undertook an application to access the data. The data analysis is further used for diagnosis. the authors suggest further enhancement of the proposed work. 
The authors in this paper [8] put forward another efficient health monitoring system involving three stages: collection, transmission and last phase is utilization. The system is equipped to gather information on the vital parameters of the body of the patient and apprise then doctor with current details. The authors have supported the work with the help of experiments by gathering the sample data belonging to patients and assess the status. The utilization stage enables the doctor to analyse and advise accordingly. Lastly, scope for further research is expressed.

The authors in this paper [9] have exemplified a system with an aim to improve the health services provided to the patients by administering networked statistics cloud enabling the doctors to diagnose the symptoms and suggest remedies. The authors aim to further enhance this system by adding more features enabling global use of the information.

The authors in the papers [10] foregrounds the discussion on one of the important aspect of IoT Health care management System, Security. The fundamental security essentials involve protection of privacy (patient identification details, confidentiality details involving the medical details related to the patient, integrity to ensure transmitted data is free of any alterations when received. authentication of the source of data, freshness of data (to avoid any replay of old data).

The authors in paper [11] probes the prevalent conditions and estimated prospects for integrating the innovative remote health care management strategies with the medicine practises. The paper also foregrounds the issues involved in the implementation of IoT services. Primarily the need for wearable sensors need to be small and light for the patients to avoid any hurdles in the mobility, and other analytics issues are studied.

\section{IoT- based Healthcare Challenges:}

Based on the literature Survey in the previous section, it can be inferred that despite great potentiality in the field of healthcare, IoT also shares some obstacles. Another challenge is Security of the data that is exchanged with other devices. Table 1. Details the challenges faced by the healthcare IoT, which includes the limits on the size and weight of sensors, the energy consumption limitation on the devices, the attacks that IoT network is susceptible to and certain analytic challenges Management of the interconnected devices along with the storage of the large amount of data collected for numerous patients is a challenging task for the hospital, it also involves analytic challenges.

Table 1. Challenges ahead of IoT Implementation.

\begin{tabular}{|c|l|}
\hline \multirow{2}{*}{$\begin{array}{c}\text { IOT } \\
\text { HEALTH CARE }\end{array}$} & Challenges \\
\cline { 2 - 2 } & $\begin{array}{l}\text { Wearable sensors limit the design of } \\
\text { sensors e.g. light, small }\end{array}$ \\
\cline { 2 - 2 } & Energy efficient design of sensors \\
\hline
\end{tabular}




\begin{tabular}{|l|l|}
\hline \multirow{4}{*}{ MANAGEMENT } & and other equipment. \\
\cline { 2 - 2 } & Security Challenges \\
\cline { 2 - 2 } & Susceptible to Attacks \\
\cline { 2 - 2 } & Analytic Challenges \\
\hline
\end{tabular}

After studying various challenges ahead of effective IoT implementation, one of the indispensable challenge is the effective use of the plethora of data generated by the healthcare managing system. This paper presents studies the role of big data in containing the problem. The next section of this paper provides a comprehensive view of big data analytics and its uses in healthcare management.

\section{Big Data Analytics:}

The phrase analytics epitomizes the method of extricating value from the data by creating and distributing reports, statistical modelling and mining, exploration and visualization of data, and related methods [12]. Big data analytics is the method of scrutinizing mammoth amount of diverse sets of data (big data) to unveil hidden sequences, relationships, market preferences and several other information. Big data Analytics can be revolutionary in healthcare by performing analysis on data and provide an abundant reference to reform many spheres of health care management such as diagnosis, understanding the side-effects of drugs, analysis related to genome etc [13]. One of the paramount importance of Big Data Analytics is Predictive analytics [14] rendering the sensed amount of data to be utilized for analyzing and predicting outcomes related to individual patients. Thus, the amount of data generated with the help of IoT in the healthcare management systems can further be used to predict various outcomes related to the prediction of diseases, the effect of medicines, etc.

\section{Conclusion and Future scope:}

The technological advancements have stimulated the growth of IoT in healthcare and have proved to be beneficial in providing efficient and low-cost healthcare services, touching every sphere of human life. Many researches have generated tremendous amount of success in improving the medical services. But it has also been observed that the IoT also deals with its own share of hurdles and certain areas still need improvements and research. This paper has brought forward some challenges faced by IoT and how Big Data Analytics can prove helpful in predictive analytics. The timely diagnosis can ameliorate many diseases. Predictive analytics, thus, will help the doctors in identifying at-risk patients. The proposed model will be implemented with the help of experimentation. 


\section{References}

1 Baranidharan T, Abipriya S, Jeyakanthaselvan C, Suganya D, Venkataprakash L. Health Monitoring using Internet of Things. IJSART. March 2016; Volume 2 (3): pp. 92-94.

2 Gupta S, Kashaudhan S, Chandra, Pandey D, Gaur PPS. IOT based Patient Health Monitoring System. International Research Journal of Engineering and Technology (IRJET). Mar 2017; Volume 4(3), pp. 2316-2319.

3 Jiménez F, and Torres R. Building an IoT-aware healthcare monitoring system., In: Proceedings of the 34th international conference of the Chilean computer science society, Santiago, Chile New York: IEEE. November 2015; 9-13, pp. 5-8.

4 Babu MVSS, et al. Smart Human Health Monitoring System by using IoT. SSRG International Journal of Electronics and Communication Engineering (ICEEMST'17) - Special Issue- March 2017.

5 Bhoomika BK, Muralidhara KN. Secured Smart Healthcare Monitoring System Based on IoT. International Journal on Recent and Innovation Trends in Computing and Communication. July 2015; volume 3(7), pp. 4958-4961.

6 Riazul Islam SL, et al. The Internet of Things for Health Care: A Comprehensive Survey. In: IEEE Access. 2015; Volume 3, pp. 678-708

7 Rajput DS, and Gaur R. An IoT Framework for Healthcare Monitoring Systems. International Journal of Computer Science and Information Security. 2016; 14 (5), pp. 451-455.

8 Devi SA, et al. Patient Health Monitoring System (PHMS) Using IoT Devices. International Journal of Computer Science \& Engineering Technology (IJCSET). March 2016; Volume 7(3).

9 Gupta P, Agrawal D, Chhabra J, Dhir PK. IoT based Smart HealthCare Kit. In: proceedings of International Conference on Computational Techniques in Information and Communication Technologies, IEEE, 2016.

10 Ahmed MU, et al. An Overview on the Internet of Things for Health Monitoring Systems, IoT Technologies for HealthCare, 2015.

11 Hassanalieragh $\mathrm{M}$, et al. Health Monitoring and Management Using Internetof-Things (IoT) Sensing with Cloud-Based Processing: Opportunities and Challenges., IEEE International Conference on Services Computing, New York, NY. 2015; pp. 285-292.

12 Grossman RL, and Siegel KP. Organizational models for big data and analytics. Journal of Organization Design, Special Issue on Big Data and Organization Design. 2014; vol. 3(1), pp. 20-25.

13 Marjani M, et al. Big IoT Data Analytics: Architecture, Opportunities, and Open Research Challenges. In IEEE Access. 2017; volume 5, pp. 5247-5261.

14 Reddy AR, and Kumar PS. Predictive Big Data Analytics in Healthcare. In: Second International Conference on Computational Intelligence \& 
Communication Technology (CICT). 2016; pp. 623-626. 